(C) 2021, The Authors. Published by Elsevier Inc. and Fass Inc. on behalf of the American Dairy Science Association ${ }^{\circledR}$. This is an open access article under the CC BY license (http://creativecommons.org/licenses/by/4.0/).

\title{
Familiarity influences social networks in dairy cows after regrouping
}

\author{
B. Foris, ${ }^{1,2} \odot$ H.-G. Haas, ${ }^{1} \odot$ J. Langbein, ${ }^{3}$ ๑) and N. Melzer ${ }^{1 *}$ () \\ ${ }^{1}$ Institute of Genetics and Biometry, Leibniz Institute for Farm Animal Biology, 18196 Dummerstorf, Mecklenburg-Western Pomerania, Germany \\ ${ }^{2}$ Animal Welfare Program, Faculty of Land and Food Systems, University of British Columbia, Vancouver, BC, V6T 1Z4, Canada \\ ${ }^{3}$ Institute of Behavioural Physiology, Leibniz Institute for Farm Animal Biology, 18196 Dummerstorf, Mecklenburg-Western Pomerania, Germany
}

\begin{abstract}
Regrouping is common practice when managing dairy cow groups, and it is known to have disruptive effects on behavior and production. The presence of a small group of familiar cows upon regrouping may provide social support and mitigate some of the negative effects. In this study we investigated (1) how regrouping affects social relationships among familiar cows and (2) if cows prefer familiar individuals over unfamiliar ones as social partners after regrouping. We used 3 established groups of cows to create 2 new groups, each containing 14 cows, using subgroups of familiar animals (i.e., 4, 6, and 4 cows) from the original groups. The new groups were similar in respect to the age, parity, and sire of cows. The frequencies of grooming and displacements were determined in the walking alley, lying stalls, and feed bunk by observing $48 \mathrm{~h}$ of continuous video before regrouping, directly after regrouping, and 1 wk later. First, social network analysis was applied to investigate the effects of regrouping on the relationships within the subgroups of familiar cows. Second, we determined if familiar cows were more or less connected than would be expected by chance (i.e., assortment), considering displacement, grooming, and feed bunk neighbor networks (derived from electronic feeder data) after regrouping. Regrouping increased the number of displacements, especially in the walking alley. Within the subgroups of familiar cows, regrouping resulted in slightly more displacements, but the network structure did not change. The frequency of grooming among familiar cows remained stable across all observation periods, and the network structure was not affected by regrouping. We found positive assortment in grooming and feed bunk neighbor networks, thereby suggesting that cows preferred familiar individuals as grooming partners and feeding neighbors directly after regrouping and, to a smaller extent, 1 wk later. The effect
\end{abstract}

Received May 14, 2020.

Accepted October 17, 2020.

*Corresponding author: melzer@fbn-dummerstorf.de of familiarity on displacements depended on the pen area. The weak assortment directly after regrouping at the feed bunk indicated that familiar cows displaced each other more than unfamiliar ones, possibly because they were neighbors more often. Our results indicated that a small group of familiar cows may provide ongoing social buffering after regrouping. Further research with multiple groups and larger group sizes is needed to determine whether similar effects are consistently present when groups of familiar cows are subjected to regrouping.

Key words: animal welfare, group management, social stress, social support

\section{INTRODUCTION}

Dairy cows are regrouped several times during a lactation according to their reproductive status, nutritional needs, and milk production (Bøe and Færevik, 2003; Chebel et al., 2016). Ample evidence exists regarding the disruptive effects of regrouping on animal welfare and performance, such as increased agonistic interactions, decreased social licking, reduced lying time, and decreased DMI and milk production (e.g., Grant and Albright, 2001; von Keyserlingk et al., 2008; Schirmann et al., 2011). The most pronounced effects of regrouping seem to decrease after approximately $3 \mathrm{~d}$ (Kondo and Hurnik, 1990; Grant and Albright, 2001), but it may take up to $2 \mathrm{wk}$ to reestablish a stable social group structure (Hasegawa et al., 1997; Bøe and Færevik, 2003; Patison et al., 2010). Frequent and unpredictable changes in the social environment can lead to social stress and increased risk of illness (Proudfoot et al., 2012; Proudfoot and Habing, 2015). Despite the known negative effects associated with regrouping, little progress has been made in developing practical solutions to improve grouping strategies on dairy farms.

It has been suggested that the presence of familiar cows may facilitate integration in the herd (Bøe and Færevik, 2003), and that familiar animals could provide social support during stressful periods (Rault, 2012). Previous studies indicate that familiarity influences agonistic and affiliative relationships, as well as the spatial 
proximity of cows. For example, heifers encountered less agonistic interactions when they were introduced into a cow herd in pairs (Neisen et al., 2009). Another study followed focal cows in a dynamic early lactation group and found that familiar pairs were often neighbors during feeding and resting, and they exhibited more grooming (Gutmann et al., 2015). Moreover, familiarity was related to closer proximity in pairs of steers (Patison et al., 2010), and dairy cow dyads with long-term familiarity (i.e., growing up together or a shared dry period) exhibited higher spatial synchronicity (Gygax et al., 2010). Using small groups of familiar cows instead of individuals as a unit for regrouping may help to mitigate some of the disruptive effects mentioned above. However, it is not known if short-term familiarity (i.e., housed in the same group before regrouping) influences the group-level structure of social interactions and the spatial associations upon regrouping. Moreover, most previous studies of the detrimental effects of disrupting the established social group structure were based on dyadic-level analysis. Social network analysis provides a framework for comprehensively assessing the effects of familiarity on social interactions and spatial associations at the group level (Farine and Whitehead, 2015; Foris et al., 2019a).

A previous study showed that agonistic interactions can be unevenly distributed between different functional areas in the pen (Foris et al., 2019a). Moreover, distinct resources may be associated with different social relationships according to the individual motivation of cows to gain access (Val-Laillet et al., 2008). In particular, differences in the frequency of agonistic interactions between pen areas are expected when the group composition changes. New dominance relationships must be established between unfamiliar cows after regrouping (Bouissou et al., 2001), which may lead to non-resource-related fights. However, it is unknown whether the effect of familiarity on agonistic interactions after regrouping depends on the functional area in the pen.

In this study, we aimed to investigate how social behavior changes when small groups of familiar cows are subjected to regrouping. First, we studied the effect of regrouping on grooming and displacement relationships among familiar cows. Second, we investigated if cows prefer familiar individuals over unfamiliar individuals as grooming, displacement, or feed bunk neighbor partners after regrouping. We hypothesized that more grooming should occur between familiar cows compared with unfamiliar cows and that familiar cows would be neighbors more often at the feed bunk. Moreover, we predicted that more displacement should occur between unfamiliar cows after regrouping, and that the influ- ence of familiarity on agonistic behavior would depend on the pen area where encounters occur.

\section{MATERIALS AND METHODS}

\section{Animals and Housing}

The study was conducted at the Leibniz Institute for Farm Animal Biology (FBN, Dummerstorf, Germany) experimental cattle facility from October to November during 2016. The facility and procedures were described in detail in a previous study (Foris et al., 2019a). Briefly, cows were housed in pens containing 15 lying stalls, an automatic brush (Happy Cow, Kerbl, Germany), 2 electronic water bins, and 10 electronic feed bins (Insentec RIC, Marknesse, the Netherlands), and they were milked twice daily. In this study, we enrolled 3 groups of lactating Holstein cows with group sizes of 12 cows (G1), 14 cows (G2), and 11 cows (G3) to create 2 new mixed groups (M1 and M2), each containing 14 cows. Groups M1 and M2 were created by choosing subgroups of 4 cows, 6 cows, and 4 cows from G1, G2, and G3 respectively. We chose cows from the original groups in a semi-randomized manner, accounting for age, parity, and sire to ensure the similarity of M1 and M2 [mean \pm SD; age (yr): M1: $4.77 \pm 0.76$, M2: $4.84 \pm 0.73$; parity: M1: $2.86 \pm 0.77$, M2: 2.71 $\pm 0.73]$. To facilitate identification, cows were marked with an individual alphanumeric sign on their shoulder and flank using hair dye. We ensured that there were no mother-daughter pairs among the observed cows.

All animal care and experimental procedures were performed in accordance with the German welfare requirements for farm animals and the $\mathrm{ASAB} / \mathrm{ABS}$ Guidelines for the Use of Animals in Research (Anonymous, 2016). All procedures involving animal handling and treatment were approved by the Committee for Animal Use and Care of the Ministry of Agriculture, Environment and Consumer Protection of the federal state of Mecklenburg-Vorpommern, Germany (Reference number: 7221.3-2-033/15).

\section{Data Collection}

We recorded the behavior of each group using 2 video cameras (Sony YC 3189, Sony Corp., Tokyo, Japan) and a digital recorder (EDR HD-2H14/4H4, EverFocus Electronics Corp., New Taipei City, Taiwan). Groups G1 to G3 were established stable groups, but cows occasionally left for a few days to participate in another experiment as social companion animals. To avoid any effect from this, we started the observations at least 7 d after the last cow was reintroduced or left the group 
(Kondo and Hurnik, 1990). For G1 to G3, we analyzed $48 \mathrm{~h}$ of continuous video (starting from midnight); data were collected on d 15 and 16 for G1, and on d 7 and 8 for G2 and G3 before regrouping (PRE), according to equipment availability. Groups M1 and M2 were created during the day (at $1400 \mathrm{~h}$ ), and we analyzed two 48-h periods for these groups: starting directly after regrouping (POST), and 1 wk later (1 WK). We only considered periods when the group was undisturbed and all cows were present, and thus $2 \mathrm{~h}$ were excluded each day on average when the cows went for milking or due to occasional short-term interruptions, such as cleaning and typical management activities. Social interactions were identified by 1 trained observer using Mangold Interact V15 (Mangold International GmbH, Arnstorf, Germany). Continuous event sampling was used to record social interactions in the complete pen area, where we recorded the actor, receiver, and location of each event. We recorded displacements as agonistic behavior (i.e., headbutt or push from the actor that resulted in the receiver leaving the feed bunk or lying stall, or taking a minimum of 2 steps in the walking alley). We recorded grooming as affiliative behavior (i.e., actor licking the receiver for a minimum of $15 \mathrm{~s}$ when both cows were in the same pen area). We distinguished between the following areas in the pen: feed bunk (feed and water bins together), lying stalls, and walking alley. The ethogram was developed in a previous study to achieve reliable detection based on the video footage (Foris et al., 2019a). The intra-observer reliability was tested based on 2 video samples (total of $5.5 \mathrm{~h}$ ) that covered different hours in the day, and the observer identified interactions with $93.5 \%$ sensitivity and $81.1 \%$ precision (see Supplemental Table S1; https://doi.org/ 10.5281/zenodo.4309739).

In addition, we used electronic feed and water bin data to detect direct neighbors at the feed bunk during the same periods considered for video analysis. The electronic bins recorded the start and end time of each visit as well as the identity of the cow. Data preparation and quality checks were performed as described by Foris et al. (2019b) (i.e., feeder visits longer than $60 \mathrm{~min}$ and drinker visits longer than 10 min were excluded). For each pair of cows, we quantified the number of events when they occupied neighboring bins at the feed bunk.

Heat detection was performed by the farm staff, and we aimed to avoid times in the analysis when cows were in heat according to insemination protocols. However, preliminary video analysis revealed that 3 cows in M2 (2 cows from G2 and 1 from G3) were influenced by previously unnoticed signs of heat during the night (i.e., chasing, mounting, and being mounted) during the $1 \mathrm{WK}$ observation period. Therefore, we also performed all of the calculations for M2 by excluding these cows and considered the corresponding results when they differed from the results obtained for the complete group.

\section{Statistical Analysis}

The analyses were performed in $\mathrm{R}$ ( $\mathrm{R}$ Core Team, 2020). The significance level was set to $P<0.05$.

Social Networks. The description of the social network terminology and definitions for the calculated measures are presented in Table 1 [see Farine and Whitehead (2015) for more details]. Briefly, in social networks, the nodes represent individual cows and the edges represent the relationships between them. In grooming or displacement networks, edges are directed from the actor toward the receiver of the interaction and the strength of a relationship is indicated by the edge weight (i.e., number of interactions between 2 cows). We produced grooming and displacement networks based on all of the observed events and also considered displacement networks separately for each pen area to distinguish between resource-related and non-resourcerelated competition.

Descriptive Analysis. We described how regrouping affected the spatial distribution and group-level structure of grooming and displacement. For each time point (i.e., PRE, POST, 1 WK), we determined the frequency of social interactions in the whole pen as well

Table 1. Terms and definitions related to social network analysis

\begin{tabular}{|c|c|}
\hline Term & Definition \\
\hline Node & Individuals in a network; each node represents a cow within the group \\
\hline Edge & Relationship between individuals in a network; social interaction or spatial association between 2 cows \\
\hline Edge direction & $\begin{array}{l}\text { Directed edges point from the actor to the receiver of a social interaction; undirected edges represent spatial association } \\
\text { between cows }\end{array}$ \\
\hline Edge weight & Number of interactions or associations between 2 cows; represented by edge width \\
\hline Density & Ratio of existing connections against all possible connections in the network \\
\hline Dyadic census & $\begin{array}{l}\text { The number of mutual (both cows in a dyad initiated interaction with the other), asymmetric ( } 1 \text { one cow in a dyad } \\
\text { initiated interaction with the other), and null (dyads with no interactions) relationships in directed networks }\end{array}$ \\
\hline Assortment & $\begin{array}{l}\text { Correlation in the phenotype of connected individuals; the assortativity coefficient shows if familiar cows are more or } \\
\text { less connected than expected by chance }\end{array}$ \\
\hline
\end{tabular}


as in each pen area (feed bunk, walking alley, and lying stalls). Moreover, we calculated corresponding social network measures such as density and dyadic census (Table 1) using the $\mathrm{R}$ package igraph (Csardi and Nepusz, 2006).

Effect of Regrouping on Social Behavior Among Familiar Cows. Following Smith et al. (2019), we considered the 6 subgroups of familiar cows (i.e., small groups chosen from G1 to G3 to create M1 and M2) as experimental units and used linear mixed models to investigate how the social networks within these subgroups changed between PRE, POST, and 1 WK periods. In the first step, we investigated the effect of regrouping on the frequency of social interactions among familiar cows. We considered period (PRE, POST, $1 \mathrm{WK}$ ) as fixed effect and subgroup, subgroup $\times$ period, cow nested in subgroup, and the residual effect as random effects. The dependent variables were frequency of initiated (1) displacements and (2) grooming within the subgroup per cow. The dependent variables were log-normal transformed, and a constant value of 1 was added to enable transformation for observed zeros. The means and $95 \%$ confidence intervals were back transformed. In the second step, we investigated the effect of regrouping on the structure of subgroup networks, focusing on density. We considered period (PRE, POST, $1 \mathrm{WK}$ ) as a fixed effect and subgroup and residual effect as random effects. The analyses were performed using the $\mathrm{R}$ package lme4 (Bates et al., 2015) by applying the lmer function, and the $95 \%$ confidence intervals were obtained using the lsmeans function from the lmerTest package (Kuznetsova et al., 2017). We applied the Tukey-Kramer test to correct for multiple testing as implemented in the $\mathrm{R}$ package lsmeans (Lenth 2016). Moreover, we used the KenwardRoger adjustment. To control for the potential effect of different subgroup sizes, we performed the same analyses with different combinations of cows randomly excluded to obtain subgroups with 4 cows only.

Effect of Familiarity on Social Networks After Regrouping. We investigated if familiarity (defined as coming from the same original group; G1-G3) influenced the social relationships between cows in M1 and M2 during the POST and 1 WK periods. To determine if familiar cows were more or less connected than they would be expected by chance (i.e., assortment) we calculated the assortativity coefficients (Farine and Whitehead, 2015) for each network during the POST and $1 \mathrm{WK}$ periods using the R package assortnet (Farine, 2016). Specifically, positive coefficients suggested that familiar cows were more connected than expected, whereas negative coefficients suggested that familiar cows were less connected than expected. To determine whether the obtained assortativity coefficients were sig- nificantly different from random, 2-tailed $P$-values were calculated based on a permutation test with 10,000 randomized networks. In addition, to test the effect of familiarity on feeding neighbor preferences, we created networks based on the electronic bin data where the edges were undirected and the strength of a relationship was represented by the number of times 2 cows were feeding next to each other.

\section{RESULTS AND DISCUSSION}

\section{Descriptive Results}

The frequency and spatial distribution of recorded social interactions during PRE, POST, and 1 WK periods are reported in Table 2. During the PRE period, the majority of grooming was observed in the lying stalls, and this pattern became more pronounced in the POST period. During the PRE period, most displacements were observed at the feed bunk, in line with previous work (Val-Laillet et al., 2008). The number of displacements increased in the POST period, and this corresponded to a higher proportion of displacements in the walking alley, with a lower proportion at the feed bunk in both groups, similar to recent findings in heifers (Nogues et al., 2020). Agonistic interactions in the walking alley were not directly associated with resource access, and thus the increase in this area was probably partly related to the formation of new dominance relationships (Kondo and Hurnik, 1990). During the 1 WK period, the spatial distribution of displacements still differed from those in the original groups, indicating that the social structure of the newly created group had not yet been established, in line with previous findings in steers (Patison et al., 2010) and dairy heifers (Hasegawa et al., 1997).

Table 3 shows the density and dyadic census for grooming and displacement networks in PRE, POST, and $1 \mathrm{WK}$ periods. The grooming networks were generally less dense (i.e., fewer connections between individuals) compared with the displacement networks. Also, the group-level structure of grooming networks changed less upon regrouping compared with displacement networks. Most existing grooming relationships were asymmetric during all 3 periods as also shown in a previous investigation (Val-Laillet et al., 2009). In contrast, most existing displacement relationships were asymmetric during the PRE period, but mutual during the POST period and also during the $1 \mathrm{WK}$ period in M1, thereby indicating the instability of the social hierarchy. In displacement networks, regrouping resulted in increased density, and more than $77 \%$ of all possible displacement relationships were present during the POST period. During the 1 WK period, the density 
Table 2. Number, percentage, and location of social interactions over $48 \mathrm{~h}$ before regrouping (PRE), directly after regrouping (POST), and 1 wk after regrouping $(1 \mathrm{WK})$

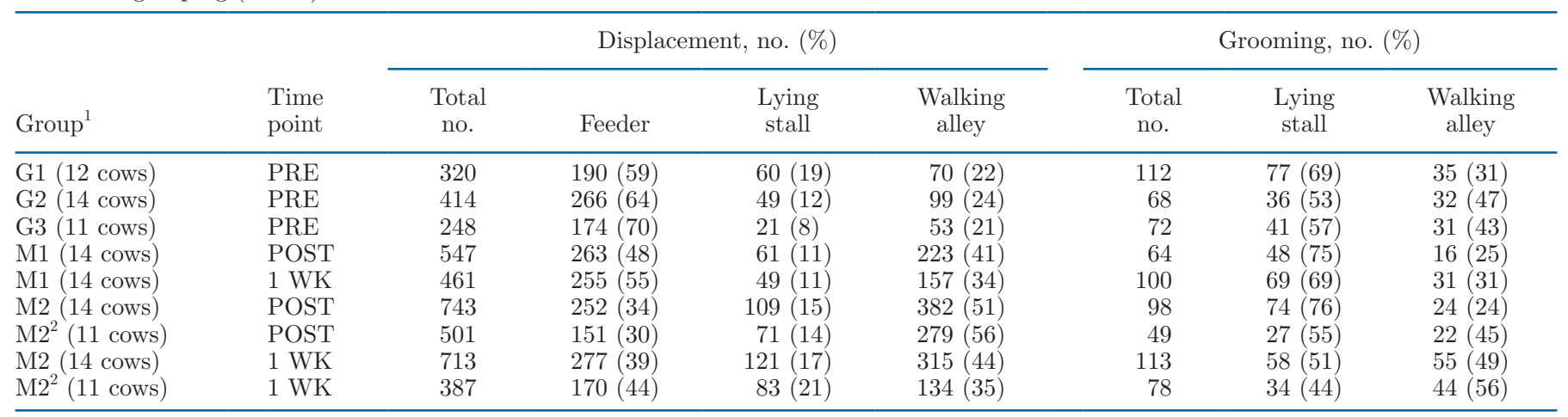

${ }^{1}$ Three groups of lactating Holstein cows with group sizes of 12 (G1), 14 (G2), and 11 (G3) cows were used to create 2 new mixed groups (M1 and M2), each containing 14 cows. Groups M1 and M2 were created by choosing subgroups of 4 cows, 6 cows, and 4 cows from G1, G2, and G3, respectively.

${ }^{2}$ The results for M2 excluding 3 cows influenced by heat during 1 WK.

increased in grooming networks alongside a decrease in displacement networks, which may have corresponded to the formation of new social bonds.

\section{Effect of Regrouping on Social Behavior Among Familiar Cows}

The differences in social behavior among familiar cows between PRE, POST, and 1 WK periods are presented in Table 4. We obtained similar findings when controlling for the differences in subgroup size, and the distribution of the adjusted $P$-values over the combinations is presented in the Supplemental Figure S1 (https://doi .org/10.5281/zenodo.4309739). Displacements among familiar cows tended to increase during the POST period compared with the PRE period. This increase was most pronounced in the walking alley, as indicated by an additional analysis of separate pen areas (Supplemental Figure S1D). This is in line with the described pattern in the overall number of displacements (Table 2 ). The number of displacements among familiar cows tended to decrease from POST to $1 \mathrm{WK}$, especially at the feed bunk (Supplemental Figure S1C), and did not differ significantly from the PRE period during the $1 \mathrm{WK}$ period. The network parameter density within the subgroups of familiar cows did not change across observation periods (Table 4). These results indicated that regrouping lead to slightly increased displacements even within subgroups of familiar cows, but this did not correspond to structural network changes in their relationships, and the effect was short lived. According to our observations, the increase in displacements among familiar cows might be a result of the increased activity in general and dominance-related fights in the walking alley.
The frequency of grooming remained relatively unchanged among familiar cows across all observation periods (Table 4). The tendency for more grooming during the $1 \mathrm{WK}$ period compared with the PRE period decreased when excluding cows affected by heat. Our results indicated that grooming behavior among familiar cows remained more stable than displacement, despite regrouping. The density in grooming networks of familiar cows did not show any change across observation periods (Table 4). Grooming has been proposed as an indicator of dyadic social bonds (Wasilewski, 2003) and to have a stress-alleviating function in receiver cows (Schmied et al., 2008; Laister et al., 2011). Our results indicated that cows did not increase grooming frequency within subgroups to deal with the stress of regrouping. This is in line with previous work that found no group-level increase in grooming when competition for food increased (Val-Laillet et al., 2009). However, the number of subgroups and the overall number of observed grooming within subgroups of familiar cows was low, which might have limited the ability to find a significant change in grooming relationships between periods. Therefore, caution should be taken when interpreting the corresponding results. Previously, in groups of similar size, we found that cows had 4 to 7 grooming partners on average (Foris et al., 2019a). Thus, having a few familiar partners may provide cows with an opportunity to maintain grooming behavior after regrouping, even when the new social bonds are not yet established. The stability of social network structure within subgroups of familiar cows implied that they may benefit from a small familiar group upon regrouping by having a known microenvironment where the outcome of social interactions is more predictable. 

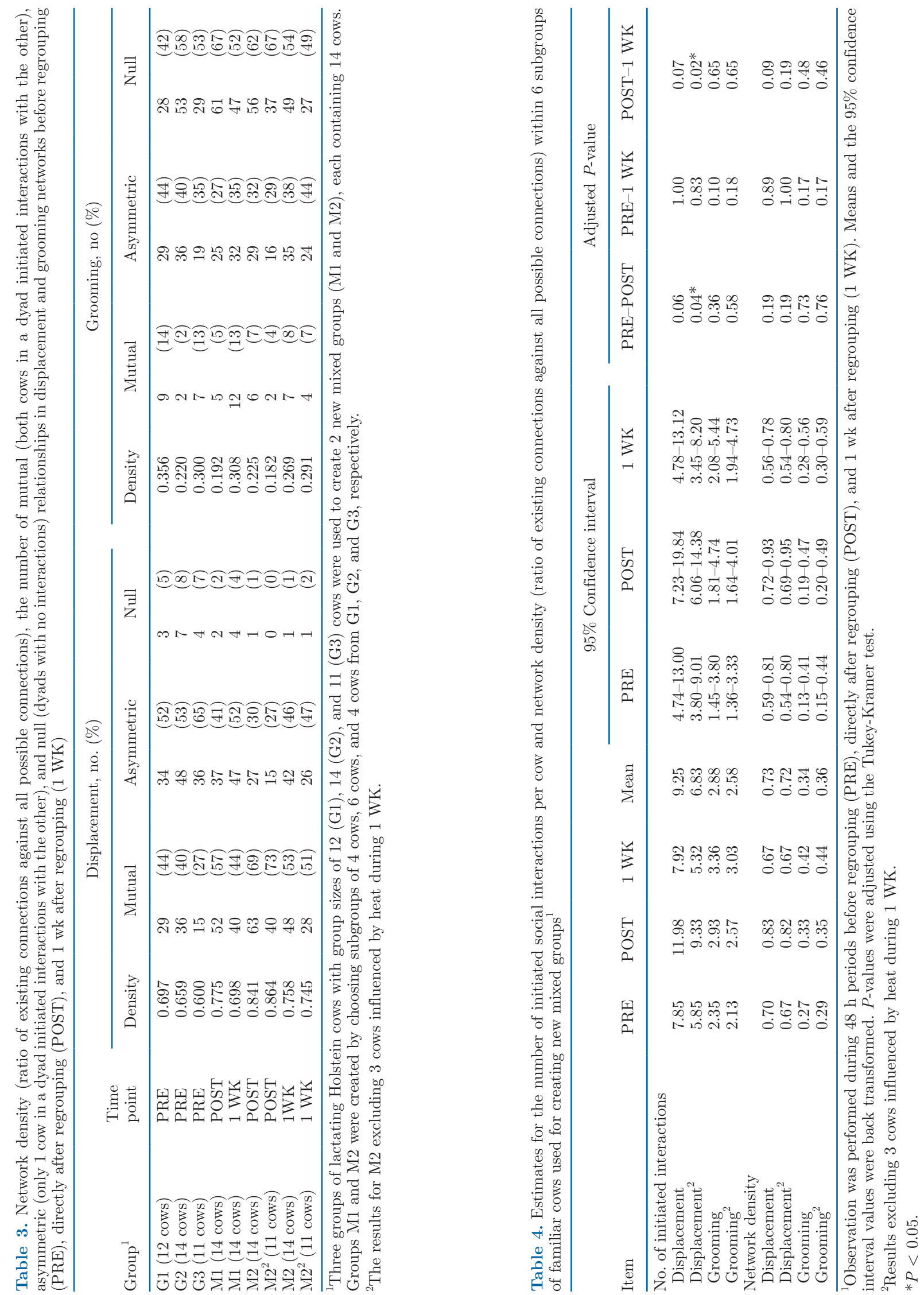


\section{Effect of Familiarity on Social Networks After Regrouping}

Figure 1 provides an example of displacement, grooming, and feed bunk neighbor networks during the POST period where assortment is visible as the relative closeness of familiar cows within each network. The assortativity coefficients for the different social network types during POST and $1 \mathrm{WK}$ periods are presented in Table 5. The positive assortativity coefficients obtained in grooming networks during the POST period indicated that cows preferred previous group mates as grooming partners. Previous studies found similar effects when investigating the influence of long-term familiarity (i.e., shared dry period or growing up together) on grooming behavior and proximity within dyads (Gygax et al., 2010; Gutmann et al., 2015). However, a recent study did not find any effect of short-term familiarity on grooming behavior when heifers were introduced into a group in pairs (Mazer et al., 2020), possibly because observations were only conducted for the first hour after regrouping. The observed positive assortment supports our finding that small groups of familiar cows maintained grooming behavior among themselves. However, this may not have been equally relevant for all individuals because some cows were not connected to their previous group members in the grooming network (see example in Figure 1). During the 1 WK period, the as- sortment was weaker, which may imply that new bonds between unfamiliar cows started to establish. When we excluded cows with signs of heat from the analysis, no assortment was found during $1 \mathrm{WK}$, possibly because the behavioral changes related to heat contributed to the effect of familiarity.

We expected the effect of familiarity on displacement networks to differ between resource-related and nonresource-related interactions. Accordingly, no effect of familiarity was observed when all pen areas were analyzed together. At the feed bunk during the POST period, familiar cows displaced each other more often than unfamiliar cows, and familiar cows were also more frequently neighbors (i.e., positive assortativity coefficients). This effect decreased or disappeared during the 1 WK period. These results may imply a higher level of behavioral synchronization between cows coming from the same group (Neisen et al., 2009; Raussi et al., 2010), and frequent displacements among familiar individuals may be a consequence of foraging close to each other. Our results supported previous findings that cows choose preferred competition partners and exhibited an increased number of agonistic interactions with their feeding neighbors (Val-Laillet et al., 2009). During the POST period, displacements in the walking alley were more common between unfamiliar cows in M1 (i.e., negative assortativity coefficients), indicating that some of the agonistic behavior in this area
A

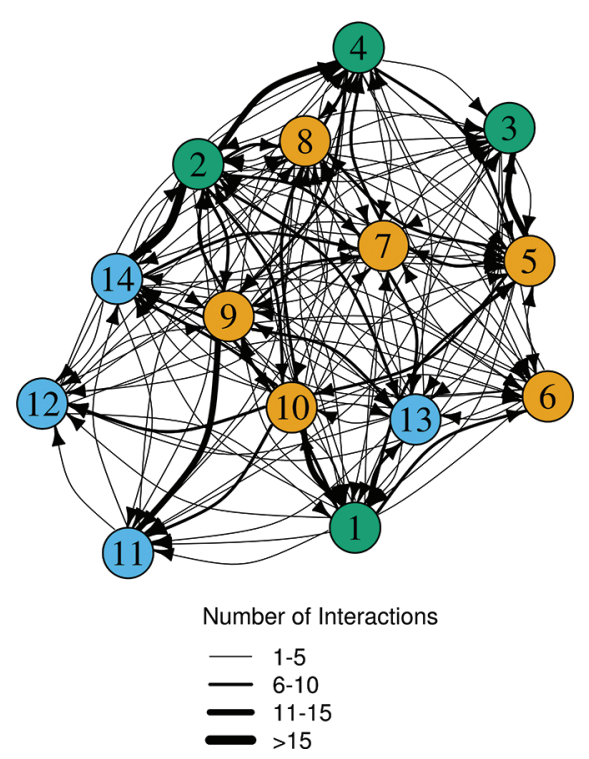

B

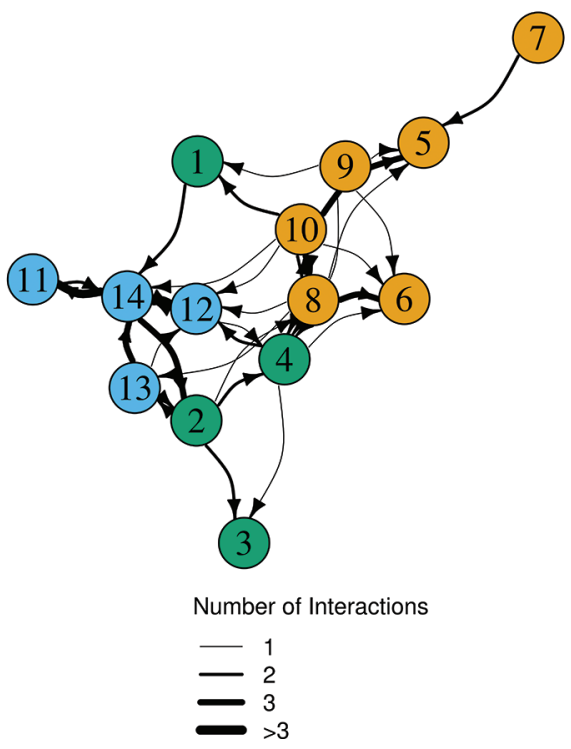

C

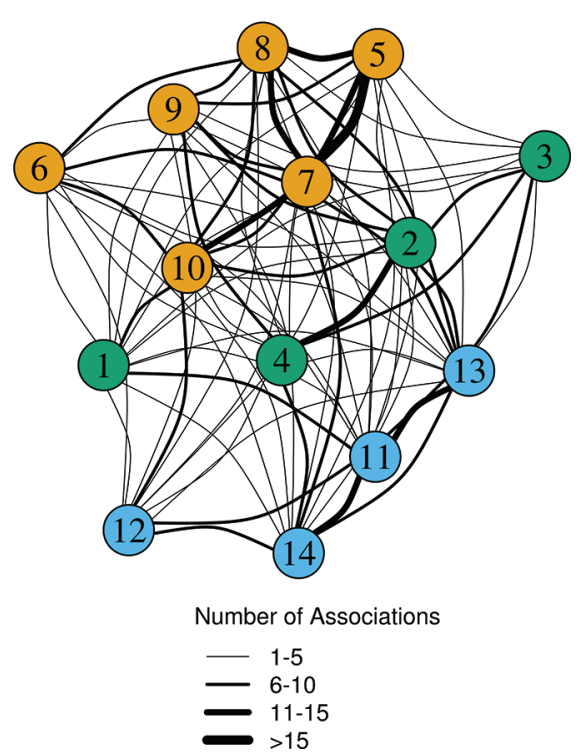

Figure 1. Exemplary social networks for (A) displacement, (B) grooming, and (C) feed bunk neighbor relationships in a group of 14 cows during $48 \mathrm{~h}$ directly after regrouping. Nodes (numbered circles) represent individual cows and the color of nodes indicates familiarity (i.e., nodes with the same color show cows coming from the same original group). Edges (lines) represent the social relationship between cows, with thicker edges indicating stronger relationships. The relative closeness of nodes with the same color in a network indicates that more connection exists between familiar cows compared with unfamiliar ones. 


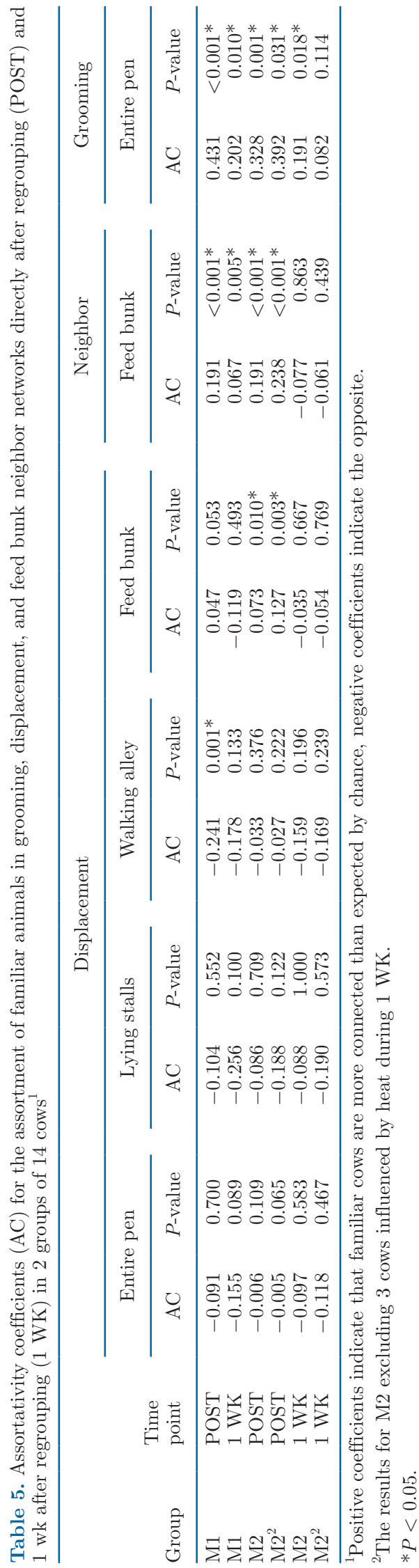

corresponded to the establishment of new dominance relationships. The absence of this pattern in M2 may indicate that familiar cows in this group had similar dominance ranks in the original groups.

\section{Limitations and Future Work}

Many studies have investigated the benefits of introducing cows into a new group in pairs (e.g., O'Connell et al., 2008; Gygax et al., 2009; Mazer et al., 2020) but 1 randomly chosen partner may not be suitable to provide social support (Rault, 2012). To the best of our knowledge, this is the first study to use small familiar groups of lactating cows to create new groups and investigate the influences on social interactions among familiar and unfamiliar cows. We defined familiarity based on group membership before regrouping, and it may be considered short-term familiarity (in a similar manner to Mazer et al., 2020). However, we cannot exclude the existence of some long-term relationships among the cows. Familiarity determined by common group membership is relatively easy to control in commercial settings, and thus it may be used as a practical parameter for regrouping. However, caution is required because group membership may be a less reliable indicator of familiarity when choosing cows from large groups. In our study, small groups were created and about one-third of the group members were familiar to each cow after regrouping. Further research is needed to determine whether similar effects are present when a small group of familiar cows is introduced into a larger group.

We performed continuous video observation, considering the entire group to obtain a detailed picture about social interactions, and coupled this with automated measurements of feed bunk neighbor relationships. This data collection method limited our ability to consider more groups due to the time-consuming nature of video observation. However, using small groups instead of individuals or pairs as units for regrouping had the advantage that we could test social network changes over time within subgroups of familiar cows. This was performed in part to overcome the limitations caused by the low number of groups in our study and to be able to investigate if the descriptive results can be statistically supported on the level of subgroups. The number of social interactions in a group is influenced by many factors (e.g., aggressiveness and sociability of cows, competition for resources, heat), and between-group variability can be expected even under similar housing conditions (Wierenga, 1990; Foris et al., 2019a). The relatively low number of groups is not uncommon in social network studies in general; in dairy cattle, the within-group variability of social interactions over time 
is not well understood due to the lack of longitudinal studies. In this study, the small number of groups and different group sizes before regrouping limited our capacity to compare the group-level social behavior across periods. These limitations may be overcome in future studies by using automated data collection methods to detect social behavior in multiple larger groups (Wurtz et al., 2019). Insights gained about the change in the spatial distribution of social interactions during the POST and $1 \mathrm{WK}$ periods may have practical relevance for automatic detection approaches. In a previous study, we showed that dominance can be reliably assessed based on automatically detected replacements in the feed bunk area (Foris et al., 2019b). However, after regrouping, feed bunk-based measures may produce misleading results due to the increased number of agonistic interactions in the walking alley. Spatial proximity is increasingly used to infer sociopositive relationships in dairy cattle (Gygax et al., 2010; Boyland et al., 2016; Rocha et al., 2020). However, our observations indicated that the proximity in the walking alley after regrouping may more likely be associated with displacement rather than grooming interactions. Thus, we suggest that the specific pen area should be considered when relying on proximity to determine social bonds in future studies.

\section{CONCLUSIONS}

Regrouping numerically increased the number of displacements in lactating cow groups, especially in the walking alley. This increase also corresponded to slightly more displacements within subgroups of familiar cows, but their relationship structure did not change. Regrouping had no effect on the grooming relationships within subgroups of familiar cows. After regrouping, cows preferred familiar individuals over unfamiliar ones as grooming partners and feeding neighbors. Displacements were more common between familiar cows at the feed bunk, where they possibly corresponded to preferred neighbors. Our results implied that a small group of familiar individuals may mitigate some of the social stress associated with regrouping. Further studies and automated data collection methods are needed to assess whether similar processes are present in large commercial groups.

\section{ACKNOWLEDGMENTS}

We thank K. D. Witt and staff members at the experimental cattle facility of the Leibniz Institute for Farm Animal Biology (FBN, Dummerstorf, Germany) for supporting our work. We thank S. Düpjan for providing cameras (Institute of Behavioural Physiology,
FBN). Furthermore, we thank G. Nürnberg and A. Tuchscherer for statistical advice (Service Group Statistical Consulting, FBN). We thank the 2 anonymous reviewers for their helpful and constructive comments that helped to improve the manuscript. This study was part of the joint project "PHÄNOMICS," which received financial support from the German Federal Ministry of Education and Research (BMBF; Grant No. 0315536G). The publication of this article was funded by the Open Access Fund of the Leibniz Institute for Farm Animal Biology (FBN). The authors have not stated any conflicts of interest.

\section{REFERENCES}

Anonymous. 2016. Guidelines for the treatment of animals in behavioural research and teaching. Anim. Behav. 111:I-IX. https://doi .org/10.1016/S0003-3472(15)00461-3.

Bates, D., M. Mächler, B. Bolker, and S. Walker. 2015. Fitting linear mixed-effects models using lme4. J. Stat. Softw. 67:1-48. https:// doi.org/10.18637/jss.v067.i01.

Bøe, K. E., and G. Færevik. 2003. Grouping and social preferences in calves, heifers and cows. Appl. Anim. Behav. Sci. 80:175-190. https://doi.org/10.1016/S0168-1591(02)00217-4.

Bouissou, M. F., A. Boissy, P. Le Neindre, and I. Veissier. 2001. The social behaviour of cattle. Pages 113-145 in Social Behaviour in Farm Animals. L. J. Keeling and H. W. Gonyou, ed. CAB International, Wallington, UK.

Boyland, N. K., D. T. Mlynski, R. James, L. J. N. Brent, and D. P. Croft. 2016. The social network structure of a dynamic group of dairy cows: From individual to group level patterns. Appl. Anim. Behav. Sci. 174:1-10. https://doi.org/10.1016/j.applanim.2015.11 .016 .

Chebel, R. C., P. R. B. Silva, M. I. Endres, M. A. Ballou, and K. L. Luchterhand. 2016. Social stressors and their effects on immunity and health of periparturient dairy cows. J. Dairy Sci. 99:32173228. https://doi.org/10.3168/jds.2015-10369.

Csardi, G., and T. Nepusz. 2006. The igraph software package for complex network research, InterJournal Complex Systems:1695. R package version 1.2.2. Accessed Aug. 17, 2020. http://igraph.org.

Farine, D. 2016. assortnet: Calculate the Assortativity Coefficient of Weighted and Binary Networks. R package version 0.12. Accessed Aug. 17, 2020. https://CRAN.R-project.org/package=assortnet

Farine, D. R., and H. Whitehead. 2015. Constructing, conducting and interpreting animal social network analysis. J. Anim. Ecol. 84:1144-1163. https://doi.org/10.1111/1365-2656.12418.

Foris, B., A. J. Thompson, M. A. G. von Keyserlingk, N. Melzer, and D. M. Weary. 2019b. Automatic detection of feeding and drinking related agonistic behavior and dominance in dairy cows. J. Dairy Sci. 102:9176-9186. https://doi.org/10.3168/jds.2019-16697.

Foris, B., M. Zebunke, J. Langbein, and N. Melzer. 2019a. Comprehensive analysis of affiliative and agonistic social networks in lactating dairy cattle groups. Appl. Anim. Behav. Sci. 210:60-67. https:// doi.org/10.1016/j.applanim.2018.10.016.

Grant, R. J., and J. L. Albright. 2001. Effect of animal grouping on feeding behavior and intake of dairy cattle. J. Dairy Sci. 84:E156E163. https://doi.org/10.3168/jds.S0022-0302(01)70210-X.

Gutmann, A. K., M. Špinka, and C. Winckler. 2015. Long-term familiarity creates preferred social partners in dairy cows. Appl. Anim. Behav. Sci. 169:1-8. https://doi.org/10.1016/j.applanim.2015.05 .007 .

Gygax, L., G. Neisen, and B. Wechsler. 2009. Differences between single and paired heifers in residency in functional areas, length of travel path, and area used throughout days 1-6 after integration into a free stall dairy herd. Appl. Anim. Behav. Sci. 120:49-55. https://doi.org/10.1016/j.applanim.2009.05.002. 
Gygax, L., G. Neisen, and B. Wechsler. 2010. Socio-spatial relationships in dairy cows. Ethology 116:10-23. https://doi.org/10.1111/ j.1439-0310.2009.01708.x.

Hasegawa, N., A. Nishiwaki, K. Sugawara, and I. Ito. 1997. The effects of social exchange between two groups of lactating primiparous heifers on milk production, dominance order, behavior and adrenocortical response. Appl. Anim. Behav. Sci. 51:15-27. https://doi .org/10.1016/S0168-1591(96)01082-9.

Kondo, S., and J. F. Hurnik. 1990. Stabilization of social hierarchy in dairy cows. Appl. Anim. Behav. Sci. 27:287-297. https://doi.org/ 10.1016/0168-1591(90)90125-W.

Kuznetsova, A., P. B. Brockhoff, and R. H. B. Christensen. 2017. lmerTest package: tests in linear mixed effects models. J. Stat. Softw. 82:1-26. https://doi.org/10.18637/jss.v082.i13.

Laister, S., B. Stockinger, A. M. Regner, K. Zenger, U. Knierim, and C. Winckler. 2011. Social licking in dairy cattle - Effects on heart rate in performers and receivers. Appl. Anim. Behav. Sci. 130:8190. https://doi.org/10.1016/j.applanim.2010.12.003.

Lenth, R.V. 2016. Least-squares means: The R package lsmeans. J. Stat. Softw. 69:1-33. https://doi.org/10.18637/jss.v069.i01.

Mazer, K. A., P. L. Knickerbocker, K. L. Kutina, and J. M. Huzzey. 2020. Changes in behavior and fecal cortisol metabolites when dairy cattle are regrouped in pairs versus individually after calving. J. Dairy Sci. 103:4681-4690. https://doi.org/10.3168/jds.2019 $-17593$.

Neisen, G., B. Wechsler, and L. Gygax. 2009. Effects of the introduction of single heifers or pairs of heifers into dairy-cow herds on the temporal and spatial associations of heifers and cows. Appl. Anim. Behav. Sci. 119:127-136. https://doi.org/10.1016/j.applanim.2009 .04 .006 .

Nogues, E., B. Lecorps, D. M. Weary, and M. A. G. von Keyserlingk. 2020. Individual variability in response to social stress in dairy heifers. Animals (Basel) 10:1440. https://doi.org/10.3390/ ani10081440.

O'Connell, N. E., H. C. F. Wicks, A. F. Carson, and M. A. McCoy. 2008. Influence of post-calving regrouping strategy on welfare and performance parameters in dairy heifers. Appl. Anim. Behav. Sci. 114:319-329. https://doi.org/10.1016/j.applanim.2008.03.004.

Patison, K. P., D. L. Swain, G. J. Bishop-Hurley, G. Robins, P. Pattison, and D. J. Reid. 2010. Changes in temporal and spatial associations between pairs of cattle during the process of familiarisation. Appl. Anim. Behav. Sci. 128:10-17. https://doi.org/10.1016/ j.applanim.2010.10.001.

Proudfoot, K., and G. Habing. 2015. Social stress as a cause of diseases in farm animals: Current knowledge and future directions. Vet. J. 206:15-21. https://doi.org/10.1016/j.tvjl.2015.05.024.

Proudfoot, K. L., D. M. Weary, and M. A. G. von Keyserlingk. 2012. Linking the social environment to illness in farm animals. Appl. Anim. Behav. Sci. 138:203-215. https://doi.org/10.1016/j applanim.2012.02.008

R Core Team. 2020. R: A language and environment for statistical computing. R Foundation for Statistical Computing, Vienna, Austria. R version 3.6.3. https://www.R-project.org.

Rault, J. L. 2012. Friends with benefits: Social support and its relevance for farm animal welfare. Appl. Anim. Behav. Sci. 136:1-14. https://doi.org/10.1016/j.applanim.2011.10.002.
Raussi, S., S. Niskanen, J. Siivonen, L. Hänninen, H. Hepola, L. Jauhiainen, and I. Veissier. 2010. The formation of preferential relationships at early age in cattle. Behav. Processes 84:726-731. https:// doi.org/10.1016/j.beproc.2010.05.005.

Rocha, L. E. C., O. Terenius, I. Veissier, B. Meunier, and P. P. Nielsen. 2020. Persistence of sociality in group dynamics of dairy cattle. Appl. Anim. Behav. Sci. 223:104921. https://doi.org/10.1016/j .applanim.2019.104921.

Schirmann, K., N. Chapinal, D. M. Weary, W. Heuwieser, and M. A. G. von Keyserlingk. 2011. Short-term effects of regrouping on behavior of prepartum dairy cows. J. Dairy Sci. 94:2312-2319. https: //doi.org/10.3168/jds.2010-3639.

Schmied, C., S. Waiblinger, T. Scharl, F. Leisch, and X. Boivin. 2008 Stroking of different body regions by a human: Effects on behaviour and heart rate of dairy cows. Appl. Anim. Behav. Sci. 109:25-38. https://doi.org/10.1016/j.applanim.2007.01.013.

Smith, L. A., D. L. Swain, G. T. Innocent, I. Nevison, and M. R. Hutchings. 2019. Considering appropriate replication in the design of animal social network studies. Sci. Rep. 9:7208. https://doi.org/ 10.1038/s41598-019-43764-9.

Val-Laillet, D., V. Guesdon, M. A. G. von Keyserlingk, A. M. de Passillé, and J. Rushen. 2009. Allogrooming in cattle: Relationships between social preferences, feeding displacements and social dominance. Appl. Anim. Behav. Sci. 116:141-149. https://doi.org/10 .1016/j.applanim.2008.08.005.

Val-Laillet, D., D. M. Veira, and M. A. G. von Keyserlingk. 2008. Short communication: Dominance in free-stall-housed dairy cattle is dependent upon resource. J. Dairy Sci. 91:3922-3926. https:// doi.org/10.3168/jds.2008-1332.

von Keyserlingk, M. A. G., D. Olenick, and D. M. Weary. 2008. Acute behavioral effects of regrouping dairy cows. J. Dairy Sci. 91:10111016. https://doi.org/10.3168/jds.2007-0532.

Wasilewski, A. 2003. "Freundschaft" bei Huftieren? - Soziopositive Beziehungen zwischen nicht-verwandten artgleichen Herdenmitgliedern. PhD thesis. Philipps-Universität Marburg, Germany.

Wierenga, H. K. 1990. Social dominance in dairy cattle and the influences of housing and management. Appl. Anim. Behav. Sci 27:201-229. https://doi.org/10.1016/0168-1591(90)90057-K.

Wurtz, K., I. Camerlink, R. B. D'Eath, A. P. Fernández, T. Norton, J. Steibel, and J. Siegford. 2019. Recording behaviour of indoorhoused farm animals automatically using machine vision technology: A systematic review. PLoS One 14:e0226669. https://doi.org/ 10.1371/journal.pone.0226669.

\section{ORCIDS}

B. Foris ๑ https://orcid.org/0000-0002-0901-3057

H.-G. Haas ๑ https://orcid.org/0000-0003-0353-4019

J. Langbein @ https://orcid.org/0000-0002-1170-5431

N. Melzer ๑ https://orcid.org/0000-0002-9586-1588 